01

\title{
Физически корректные конфигурационные пространства в описании внутренней динамики жесткой молекулы
}

\author{
(C) А.В. Буренин \\ Институт прикладной фризики РАН, \\ 603950 Нижний Новгород, Россия \\ e-mail: buran@appl.sci-nnov.ru
}

Поступила в редакцию 18.04.2019 г.

В окончательной редакции 03.07.2019 г.

Принята к публикации 08.07.2019 г.

\begin{abstract}
Рассмотрено семейство конфигурационных пространств в описании внутренней динамики жесткой молекулы. Показано, что требование физической корректности описания приводит к серьезным ограничениям для таких пространств. В частности, для нелинейной жесткой молекулы допустимы только пространства, удовлетворяющие условию Эккарта.
\end{abstract}

Ключевые слова: колебание, вращение, условия Эккарта и Сейвеца.

DOI: $10.21883 /$ OS.2019.11.48506.144-19

\section{Введение}

При описании изолированных атомов и молекул в хорошем приближении можно пренебречь вкладами, связанными со спинами электронов и ядер (называемыми тонкими и сверхтонкими взаимодействиями [1]). Координатный гамильтониан записывается в виде

$$
H=T_{\text {эл }}+T_{\text {яд }}+V_{\text {эл-эл }}+V_{\text {яд-яд }}+V_{\text {эл-яд }},
$$

где $T_{\text {эл }}$ и $T_{\text {яд }}-$ кинетические энергии электронов и ядер, а $V_{\text {эл-эл }}, V_{\text {яд-яд }}$ и $V_{\text {эл-яд }}-$ энергии кулоновского взаимодействия электронов, ядер и электронов с ядрами. При этом внутренние динамики атомных и молекулярных систем различаются качественно [2]. Действительно, характерным признаком молекулярной системы является наличие вращательного движения системы как целого, а атомной системы - его отсутствие. Физически корректным исходным приближением для описания такого движения является модель свободного вращения твердого тела [1]. Это означает, что молекулярная система представляет собой некоторую структуру (микрокристалл) с коллективными движениями частиц. В результате конфигурационные пространства атома и молекулы должны серьезно различаться. В атоме хорошим исходным приближением является описание движения каждого электрона в отдельности в усредненном поле, созданном ядром и остальными электронами. То есть полное движение есть совокупность одночастичных движений, и поэтому прямая сумма их конфигурационных пространств образует полное конфигурационное пространство. При наличии коллективных движений строить так конфигурационное пространство нельзя принципиально. Задача его формирования весьма нетривиальна. Для ее решения необходимо дать определения отдельных типов коллективных движений (в том числе вращательного движения). Причем требуется учесть, что структура молекулы характеризуется неявной геометрической группой симметрии [2]. Само понятие данной структуры удается ввести лишь на основе приближения БорнаОппенгеймера (БО). При этом неявная симметрия заменяется аналогичной ей явной симметрией. Только после этого можно математически корректно поставить саму задачу нахождения дискретного спектра молекулы. Таким образом, физически правильное конфигурационное пространство коллективных движений молекулы формируется отдельно в каждом электронном состоянии.

В настоящей работе проводится анализ конфигурационных пространств в описании внутренней динамики жесткой молекулы. Показано, что требование физической корректности описания приводит к серьезным ограничениям для таких пространств. В частности, для нелинейной жесткой молекулы допустимы только пространства, удовлетворяющие условию Эккарта.

\section{Определение движений жесткой молекулы}

Приближение БО [3-5] основано на сильном различии в массах электронов и ядер:

$$
m_{\text {эл }} \ll M_{\text {яд }} \text {. }
$$

Условие (2) позволяет считать ядра медленной подсистемой, а электроны - быстрой. То есть состояние электронной подсистемы адиабатически следит за состоянием ядерной подсистемы. Поэтому сначала решается задача о движении электронов в поле неподвижных ядер 
при произвольной их конфигурации:

$$
\begin{aligned}
\left(T_{\text {эл }}\right. & +V_{\text {эл-эл }}+V_{\text {яд-яд }} \\
& \left.+V_{\text {эл-яд }}\right) \Psi_{\text {эл }}^{(n)}=E_{\text {эл }}^{(n)} \Psi_{\text {эл }}^{(n)},
\end{aligned}
$$

где индекс $n$ задает совокупность электронных квантовых чисел. Далее в заданном электронном состоянии решается задача о движении ядер с эффективным потенциалом взаимодействия $E_{\text {эл }}^{(n)}\left(X_{\text {яд }}\right)$, по определению зависящим от мгновенной ядерной конфигурации:

$$
\left[T_{\text {яд }}+E_{\text {эл }}^{(n)}\left(X_{\text {яд }}\right)\right] \Phi_{\text {яд }}^{(n, v)}=E_{\text {яд }}^{(n, v)} \Phi_{\text {яд }}^{(n, v)},
$$

где индекс $v$ задает совокупность ядерных квантовых чисел. С качественной точки зрения молекулы делятся на жесткие и нежесткие. Для жестких молекул в невырожденных электронных состояниях эффективный потенциал имеет один минимум, а в нежестких молекулах необходимо учитывать несколько минимумов, так как внутреннее движение включает переходы между ними. Фактически уравнение (4) не задано и остается чисто формальным, пока не введено правильное конфигурационное пространство. При этом кинетическая и потенциальная части эффективного ядерного гамильтониана в переменных этого пространства должны быть инвариантными для преобразований геометрической группы, которая для жесткой молекулы совпадает с точечной группой ее единственной равновесной конфигурации. Тем самым для одного электронного состояния делается переход от неявной симметрии к явной [2].

Для введения физически корректных понятий поступательного, вращательного и колебательного движений жесткой молекулы воспользуемся тем, что форма эффективного потенциала является твердым телом. Действительно эффективный потенциал можно только сдвигать как целое и вращать как целое. Поэтому под поступательным движением будем понимать сдвиг эффективного потенциала, а под вращательным движением - вращение эффективного потенциала вокруг оси, проходящей через центр масс молекулярной системы, при неизменном положении ядер в этом потенциале. Так как ядра не смещаются в эффективном потенциале, то определенные таким образом поступательное и вращательное движения свободны, что и требуется для однородного и изотропного пространства. Соответственно под колебательным движением будем понимать смещение ядер в эффективном потенциале при неизменном положении этого потенциала в пространстве. Для описания введенных так внутренних движений используем декартовы системы координат с началом в центре масс молекулярной системы, что позволяет отделить поступательное движение. Введем неподвижную систему координат (НСК) с ориентацией осей, фиксированной в пространстве, и подвижную систему координат (ПСК) с ориентацией осей, фиксированной относительно эффективного потенциала. Ориентация ПСК относительно НСК задается тремя углами Эйлера. В итоге изменение ориентации ПСК при сохранении в ней координат ядер описывает свободное вращательное движение, а изменение координат ядер в ПСК при сохранении ее ориентации - колебательное движение в эффективном потенциале.

Важнейшим условием приведенного физически корректного определения вращательного и колебательного движений является фиксация осей ПСК относительно эффективного потенциала, являющегося твердым телом. Для нелинейной молекулы как твердое тело можно также выбрать равновесную конфигурацию, поскольку в этом случае пространственные положения эффективного потенциала и равновесной конфигурации жестко связаны. Отказ от данного рецепта фиксации приводит к очень серьезным проблемам вследствие возникновения поворотов ПСК, обусловленных смещением ядер в эффективном потенциале. В качестве примера рассмотрим приведенный в [6] выбор осей ПСК вдоль главных осей мгновенного эллипсоида инерции молекулы. Легко понять, что ориентация главных осей может меняться при колебательном смещении ядер в эффективном потенциале. Это ведет к повороту осей ПСК, причем на такой поворот действует поле и соответствующее вращательное движение перестает быть свободным. Более того, аналогичный поворот всегда можно получить и путем свободного вращения с фиксированными положениями ядер в эффективном потенциале. Таким образом, поле в пространстве углов Эйлера при заданном повороте меняется в зависимости от того, каким образом реализуется этот поворот. Это означает, что такое поле не потенциально. Естественно, что оставшаяся часть поля для колебательных движений также не потенциальна. Тем не менее в последние несколько десятилетий появилось достаточно много работ с попытками упростить описание спектра нелинейной молекулы путем отказа от ПСК, фиксированной относительно ее равновесной конфигурации [7-9]. При этом вообще не учитывалось, что в этом случае принципиально меняются свойства внутренних движений. Внимание на это было обращено относительно недавно [2,10].

После сделанных определений нетрудно для заданных вращательных и колебательных смещений ядер получить их полные смещения. Но при описании внутренней динамики требуется решение обратной задачи. А именно для произвольного смещения ядер необходимо указать вращательную и колебательную части. Для однозначного решения этой задачи необходимо дополнительное условие, определяющее поворот ПСК при заданном смещении ядер ${ }^{1}$.

\footnotetext{
${ }^{1}$ Можно даже любое смещение ядер считать чисто колебательным. Однако сразу понятно, что такая картина внутреннего движения не согласуется с реальной.
} 


\section{Разделение смещений в нелинейной молекуле}

В нелинейной жесткой молекуле задача разделения полных смещений ядер на вращательные и колебательные части при фиксации ПСК относительно равновесной конфигурации впервые рассматривалась Эккартом [11]. В качестве дополнительного условия использовалось требование минимальности кинетического взаимодействия получаемых внутренних движений. В результате при смещениях ядер из равновесных положений $\mathbf{r}_{0}^{(a)}$ в положения $\mathbf{r}^{(a)}(a-$ индекс ядра) углы Эйлера, определяющие происходящий при этом поворот ПСК, должны удовлетворять условию Эккарта:

$$
\sum_{a} M_{a} \mathbf{r}_{0}^{(a)} \times \mathbf{r}^{(a)}=0
$$

где $M_{a}-$ масса ядра $a$. Важно, что условие (5) минимальности взаимодействия применимо не к любым ПСК, а только к их довольно узкому классу с фиксацией относительно твердого тела. Поэтому неправомерно утверждение [6], что отказ от такой фиксации обязательно ведет к росту взаимодействия внутренних движений из-за нарушения условия Эккарта ${ }^{2}$. Однако в [6] полагается, что такой подход все равно предпочтителен для малых многоатомных молекул из-за упрощения выражения для кинетической части колебательновращательного гамильтониана. Но при этом игнорируется, что вращательное движение в этом случае уже не свободно. Причем это намного более принципиальный недостаток по сравнению с возможным увеличением взаимодействия внутренних движений, так как он ведет к потере физической корректности получаемого описания.

Условие Эккарта задает угол поворота для ПСК с фиксацией относительно равновесной конфигурации, что приводит к определенному разделению полного ядерного движения на колебательное и вращательное. Другой выбор поворота приведет к изменению этого разделения, причем вращательное движение остается свободным. Возникает естественный вопрос, имеются ли ограничения на такой выбор. Для ответа на него рассмотрим условие выбора, использованное в $[4,10]$. Пусть ядра совершают малые смещения $\delta \mathbf{r}^{(a)}$ из какойто исходной конфигурации к некоторой близкой ей конечной. В качестве системы координат выберем НСК исходной конфигурации. Изменение положения равновесной конфигурации можно задать как смещения $\delta \mathbf{r}_{0}^{(a)}$ равновесных положений ядер:

$$
\delta \mathbf{r}_{0}^{(a)}=\delta \mathbf{r}+d \boldsymbol{\varphi} \times \mathbf{r}_{0}^{(a)},
$$

где $\delta \mathbf{r}$ - поступательное смещение центра масс, а $d \varphi$ - вектор поворота относительно оси, проходящей

\footnotetext{
2 Отметим в связи с этим, что в [9] при отказе от фиксации ПСК относительно твердого тела построено описание, в котором кинетическое колебательно-вращательное взаимодействие отсутствует полностью.
}

через центр масс. Смещения (6) точек твердого тела выберем так, чтобы они наилучшим образом отображали смещения $\delta \mathbf{r}^{(a)}$ :

$$
\begin{aligned}
\sum_{a} M_{a}\left(\delta \mathbf{r}^{(a)}-\delta \mathbf{r}_{0}^{(a)}\right)^{2}= \\
\quad=\sum_{a} M_{a}\left(\delta \mathbf{r}^{(a)}-\delta \mathbf{r}-d \boldsymbol{\varphi} \times \mathbf{r}_{0}^{(a)}\right)^{2}=\min .
\end{aligned}
$$

Из равенства нулю первых вариаций квадратичной формы $\delta \mathbf{r}$ по $d \varphi$ и получаем оптимальные значения поступательного и вращательного смещений. Оставшаяся часть смещений $\delta \mathbf{r}^{(a)}$ относится к колебательному типу. Для ПСК должны выполняться требования

$$
\delta \mathbf{r}_{\mathrm{opt}}=0, \quad d \boldsymbol{\varphi}_{\mathrm{opt}}=0 .
$$

Первое из них приводит к условию

$$
\sum_{a} M_{a} \delta \mathbf{r}^{(a)}=0
$$

То есть при движении ядер ПСК должна оставаться в центре масс, что соответствует правильному отделению поступательного движения. Из (9) понятно, почему в качестве весов в квадратичной форме (7) используются массы ядер (иначе ПСК не будет оставаться в центре масс). Второе требование в (8) приводит к условию Эккарта (5) с точностью до замены $\mathbf{r}^{(a)}$ на $\delta \mathbf{r}^{(a)}$. Данный вывод сделан для малых смещений $\delta \mathbf{r}^{(a)}$. Однако можно показать [4], что он справедлив и для конечных смещений $\mathbf{r}^{(a)}$.

Таким образом, принцип (7) в максимально возможной степени относит смещения ядер к свободным поступательному и вращательному движениям. Поэтому нарушение условия Эккарта означает, что к свободному вращательному движению ядер относится только часть возможного поворота системы как целого. Смещение, соответствующее оставшейся части такого поворота, заменяется дополнительным колебательным смещением в эффективном потенциале. Данная интерпретация условия Эккарта позволяет с помощью принципов симметрии показать, что только удовлетворяющий этому условию выбор ПСК является физически корректным.

В заданном электронном состоянии молекулы кинетическая и потенциальная части колебательно-вращательного гамильтониана инвариантны относительно преобразований геометрической группы. Для жесткой молекулы такая группа совпадает с точечной группой ее равновесной конфигурации [1,2]. В частности, для жесткой молекулы аммиака $\mathrm{NH}_{3}$ в основном электронном состоянии это группа $C_{3 v}$. Пусть ядра молекулы находятся в положениях равновесия. Тогда $\mathbf{r}^{(a)}=\mathbf{r}_{0}^{(a)}$, и условие Эккарта (5) выполняется. Понятно, что квадратичная форма

$$
\sum_{a} M_{a}\left(\mathbf{r}^{(a)}-\mathbf{r}_{0}^{(a)}\right)^{2}
$$


обращается в нуль, что отвечает ее минимуму. Предположим, что ядра совершают движение, отвечающее преобразованию $C_{3}^{1}$ группы $C_{3 v}$. Тогда ядро $\mathrm{H}_{1}$ переходит на место ядра $\mathrm{H}_{2}$, ядро $\mathrm{H}_{2}$ на место ядра $\mathrm{H}_{3}$, а ядро $\mathrm{H}_{3}$ на место ядра $\mathrm{H}_{1}$. Поскольку такое преобразование эквивалентно пространственной перестановке тождественных ядер, то оно является элементом симметрии гамильтониана $^{3}$. Данное движение соответствует повороту ПСК на угол $2 \pi / 3$. Так как ядра снова находятся в положениях равновесия, то квадратичная форма (10) обращается в нуль, что отвечает ее минимуму. То есть поворот ПСК удовлетворяет условию Эккарта и преобразование $C_{3}^{1}$ является чисто вращательным элементом симметрии гамильтониана. Именно с такой интерпретацией он применяется в анализе свойств симметрии внутренней динамики молекулы [1,2], причем выводы согласуются с экспериментальными данными. В случае отказа от условия Эккарта указанное движение и, как следствие, преобразование $C_{3}^{1}$ не будет чисто вращательным, так как дополняется некоторым смещением ядер в эффективном потенциале. Однако при такой интерпретации преобразования $C_{3}^{1}$ невозможен анализ свойств симметрии внутренней динамики, соответствующий экспериментальным данным.

Наконец, важно, что в заданном электронном состоянии понятие эффективного колебательно-вращательного гамильтониана, включающего эффективный потенциал взаимодействия ядер, сохраняется и при учете поправок к приближению БО [12]. Поэтому сохраняются и все сделанные здесь выводы о выборе ПСК.

Итак, единственный физически корректный выбор поворота ПСК при заданном движении ядер задается условием Эккарта. При этом любой мгновенной конфигурации ядер однозначно ставятся в соответствие как положение ПСК, так и смещения ядер от положения равновесия.

\section{Разделение смещений в линейной и квазилинейной молекуле}

Использование принципа (7) для линейных молекул приводит к некоторым особенностям [4]. Одно из трех уравнений для трех скалярных условий Эккарта (5) становится тождеством. Два других уравнения приводят к условиям для ПСК, впервые полученных Сэйвецем [13] из требования минимальности кинетического колебательно-вращательного взаимодействия в линейной молекуле. Из этих условий получаем два угла, задающих ориентацию линейной равновесной конфигурации. Третий угол Эйлера, задающий поворот вокруг такой конфигурации, остается произвольным. Эта неопределенность легко устраняется при фиксации ПСК

\footnotetext{
${ }^{3}$ В [2] показано, что преобразования точечной группы эквивалентны перестановкам тождественных ядер для любой конфигурации ядер.Но в этой работе достаточно рассмотреть только равновесную конфигурацию.
}

относительно эффективного потенциала [2], так как этот потенциал остается нелинейным. Требуется выяснить, какие условия такая фиксация дополнительно накладывает на ПСК.

Линейные молекулы отвечают непрерывным точечным группам $C_{\infty v}$ и $D_{\infty h}=C_{\infty v} \times C_{1}$. Появление непрерывной точечной группы вместо конечной точечной группы для нелинейной молекулы говорит о серьезном качественном изменении внутренней динамики при переходе к линейной молекуле. Действительно, конечные точечные группы нелинейной молекулы ведут к разбиению $3+(3 n-6)$ на вращательные и колебательные степени свободы, в то время как указанные непрерывные группы - к разбиению $2+(3 n-5)$, где $n-$ число ядер молекулы [1]. Анализ методами симметрии на основе цепочки групп показывает [2], что все допустимые координатные мультиплеты линейных молекул (полные координатные функции стационарных состояний) принадлежат единичному представлению группы $C_{\infty v}$. Для таких координатных мультиплетов выполняется соотношение

$$
k=l+\lambda
$$

где $k, l$ и $\lambda-$ квантовые числа проекций полного внутреннего, колебательного и электронного моментов на ось молекулы. Условие (11) задает оставшийся произвольным угол Эйлера. Данное условие отвечает тому известному факту, что проекция углового момента на ось линейной молекулы обусловлена только электронноколебательным движением [1]. В невырожденных электронных состояниях $\lambda=0$.

Важно, что при таком выборе ПСК компоненты в ней полного координатного, колебательного и электронного угловых моментов удовлетворяют правильным соотношениям коммутации, аналогичным случаю нелинейной молекулы [2]:

$$
\begin{gathered}
{\left[J_{j}, J_{i}\right]=-i \varepsilon_{j i k} J_{k},} \\
{\left[l_{j}, l_{i}\right]=i \varepsilon_{j i k} l_{k}, \quad\left[\Lambda_{j}, \Lambda_{i}\right]=i \varepsilon_{j i k} \Lambda_{k},} \\
{\left[J_{j}, l_{i}\right]=0, \quad\left[J_{j}, \Lambda_{i}\right]=0,}
\end{gathered}
$$

где $J_{i}, l_{i}$ и $\Lambda_{i}$ - компоненты полного углового, колебательного и электронного угловых моментов в ПСК. В связи с этим заметим, что анализ внутренней динамики линейной молекулы традиционными методами симметрии на основе CNPI-группы приводит к серьезным трудностям [6] в связи с невозможностью однозначно зафиксировать ПСК относительно линейной конфигурации. Поэтому очень громоздкий анализ разбивается на два этапа. На первом этапе из трех углов Эйлера независимыми считаются только два. Третий угол, задающий вращение вокруг оси молекулы, обычно выбирают постоянным (наиболее общим считается выбор, в котором третий угол является функцией первых двух). Из-за отсутствия независимого третьего угла описание сильно искажается. В частности, совершенно неправильны коммутационные соотношения компонент в ПСК 
полного координатного, колебательного и электронного угловых моментов. Поэтому на втором этапе делается переход к так называемому изоморфному гамильтониану путем формального введения дополнительной степени свободы, задаваемой третьим углом Эйлера. Это позволяет убрать искажения, полученные на первом этапе.

Аналогично случаю нелинейной молекулы, используя свойство минимальности квадратичной формы (10) для являющихся элементами симметрии конечных поворотов, имеем, что единственный физически корректный выбор поворота ПСК при заданном движении ядер задается условиями Сэйвеца и условием (11). При этом произвольной мгновенной конфигурации ядер однозначно ставятся в соответствие как положение ПСК, так и смещения ядер от положения равновесия.

Квазилинейные молекулы имеют нелинейную равновесную конфигурацию. Однако их характерной особенностью является наличие нежестких переходов через барьер, вершине которого соответствует линейная конфигурация. Классическим примером является молекула воды $\mathrm{H}_{2} \mathrm{O}$ в основном электронном состоянии. Равновесная конфигурация нелинейная и отвечает точечной группе $C_{2 v}$ [1]. Имеются многочисленные экспериментальные данные для колебательных возбуждений угла между валентными связями $\mathrm{OH}$, описание которых требует учета нежестких переходов через линейную конфигурацию [14]. Само наличие таких переходов целиком связано с тем, что равновесная конфигурация искажена по сравнению с линейной конфигурацией, отвечающей аксиальной группе $D_{\infty \nu}$. Поэтому переходы ведут к восстановлению аксиальной симметрии внутренней динамики [2]. Существенно, что при этом не происходит расщепления уровней энергии, так как конечная конфигурация нежесткого перехода может быть также получена из исходной свободным вращением молекулы как целого на угол вокруг оси линеаризации. Роль нежестких переходов сводится здесь к переопределению конфигурационного пространства. В итоге для квазилинейной молекулы физически корректное конфигурационное пространство должно строиться аналогично линейной молекуле. Это принципиальное требование. Попытки использования конфигурационного пространства нелинейной молекулы (например, [6]) ведут к появлению сингулярностей в эффективном ядерном гамильтониане при прохождении оси линеаризации, поскольку обращается в нуль момент инерции относительно этой оси.

\section{Заключение}

Итак, важнейшим требованием в формировании физически корректного конфигурационного пространства жесткой молекулы в заданном электронном состоянии является фиксация ПСК относительно эффективного потенциала взаимодействия ядер, форма которого в этом состоянии является твердым телом. Для нелинейной жесткой молекулы это эквивалентно требованию фиксации ПСК относительно ее равновесной конфигурации. Отказ от данного рецепта фиксации приводит к очень серьезным проблемам вследствие возникновения поворотов ПСК, обусловленных смещением ядер в эффективном потенциале. При построении описания внутренней динамики для произвольного смещения ядер необходимо также указать вращательную и колебательную части. Для решения этой задачи требуется дополнительное условие, определяющее при произвольном смещении ядер поворот ПСК, фиксированной относительно твердого тела. Единственный физически корректный выбор такого поворота задается для нелинейной молекулы условием Эккарта, а для линейной и квазилинейной молекулы - условиями Сэйвеца и условием, что проекция углового момента на ось линейной молекулы задается только электронно-колебательным движением. При этом любой мгновенной конфигурации ядер однозначно ставятся в соответствие как положение ПСК, так и смещения ядер от положений равновесия.

\section{Финансирование работы}

Работа выполнена в рамках государственного задания Института прикладной физики РАН, проект № 00352014-0006.

\section{Конфликт интересов}

Автор заявляет, что у него нет конфликта интересов.

\section{Список литературы}

[1] Ландау Л.Д., Лифииц Е.М. Квантовая механика. М.: Наука, 1989. $767 \mathrm{c}$.

[2] Буренин А.В. Симметрия квантовой внутримолекулярной динамики. 3-е изд. Н. Новгород: ИПФ РАН, 2012. 416 с.; Burenin A.V. Symmetry of intramolecular quantum dynamics. Berlin/Boston: Walter de Gruyter, 2012. 430 p.

[3] Давыдов А.С. Квантовая механика. М.: Наука, 1973. 704 с.

[4] Браун П.А., Киселев А.А. Введение в теорию молекулярных спектров. Л.: ЛГУ, 1983. 232 с.

[5] Берсукер И.Б., Полингер В.З. Вибронные взаимодействия в молекулах и кристаллах. М.: Наука, 1983. 336 с.

[6] Bunker P.R., Jensen P. Molecular symmetry and spectroscopy. Ottawa: NRC Research Press, 1998. 750 p. Перевод: Банкер Ф., Йенсен П. Симметрия молекул и спектроскопия. М.: Мир, 2004. 764 с.

[7] Polyansky O.L., Tennyson J. // J. Chem. Phys. 1999. V. 110. P. 5056.

[8] Polyansky O.L., Graszar A.G., Shirin S.V., Zobov N.F., Barletta P., Tennyson J., Schwenke D.W., Knowles P.J. // Science. 2003. V. 299. P. 539.

[9] Грибов Л.А., Баранов В.И., Зеленщов Д.Ю. Электронноколебательные спектры многоатомных молекул. М.: Наука, 1997. $477 \mathrm{c}$.

[10] Буренин А.В. // Опт. и спектр. 2017. Т. 123. В. 4. С. 667. 
[11] Eckart C. // Phys. Rev. 1935. V. 47. P. 552.

[12] Макушкин Ю.С., Тютерев В.Г. Методы теории возмущений и эффективные гамильтонианы в молекулярной спектроскопии. Новосибирск: Наука, 1984. 240 с.

[13] Sayvetz A. // J. Chem. Phys. 1939. V. 7. P. 383.

[14] Быков А.Д., Синица Л.Н., Стариков В.И. Экспериментальные и теоретические методы в спектроскопии молекул водяного пара. Новосибирск: Изд-во СО РАН, 1999. 376 с. 